\title{
THE ADOMIAN DECOMPOSITION METHOD AND THE DIFFERENTIAL EQUATION
}

\author{
Sonia Viswam \\ $\mathrm{PhD}$ Scholar in Financial Management \\ Department of Business Studies \\ Central University of Karnataka, Gulbarga- 585367
}

\begin{abstract}
The Adomian Decomposition Method has wide spread applicability in the field of thermodynamics and other situations where there are bounded variables or constraints. They will help to decrease the monotonous and lengthy calculations. But they are not applicable to divergent situations. But their uses increase due to the convergent solutions made possible in a stochastic situation also where small changes in the inputs can lead to widespread changes in the outputs.
\end{abstract}

Key words: Stochastic, differential equations, delay equations, variable iteration method

\section{INTRODUCTION}

The solution for the coupled and non-coupled linear equations and partial differential equations are always time consuming. But the introduction of the Adomian Decomposition Method will decrease the monotony related to the process. The paper explores into the different possibilities of the Adomian Decomposition Method. This proves to be more helpful when the method is used along with other methods like Variable Iteration Method. Also, it is very helpful in solving several complex sets of equations which are non-linear and linear homogenous and non- homogenous etc. This will be also useful in the case of the equations which have co-efficient which are constant as well varying.

\section{LITERATURE REVIEW}

The researcher presents the method of solving nonlinear differential equations. The traditional methods for this are time consuming and involves the process discretisation. There is already a method called the Adomian Decomposition Method for this. But this method involves the inversion of the linear differential operator. So, in this condition the Taylor equation is used here. The solution of the problem is assumed to be $\mathrm{u}(\mathrm{x})$. and the solution is approximated on $[\mathrm{a}, \mathrm{b}]$ and the solution of the problem is such that $\mathrm{u}^{\wedge} \mathrm{n} x(0) / \mathrm{n}$ ! In this case the Taylor Polynomial Degree is the $N_{-}+m$ at $X=X(0)$ The Alienor problem converts the single variable problem into a problem with single variable which constitutes the alpha dense curves. (Cherruault and Mora ,2005)

In this research the researcher finds out the solution of the partial Schrodinger equation. In this equation by traditional methods only deal with the coefficients with the constant functions. The researchers like Dal (2009) and Ashyalyev (2009) has solved them by making use of the finite difference method. It is solved for a particular space at time t. For that purpose, the fractional Schrodinger equation which is unidimensional and multidimensional conditions are considered. The unidimensional Schrodinger equation is determined by the local boundary conditions and the multidimensional Schrodinger equation is ruled by the Dirichlet condition is considered. And the solution is made. (Asyralyev and Hicdurmaz, ,2011)

In the context of the Integro-Differential Equations it is found that the collocation method is used and the iterated collocation method will be used in this. The orthogonal functions are used to represent the time-based functions and they are applied in the context of the dynamic systems. At first all the truncated time-based variables are integrated and through the $\mathrm{P}$ operation of the matrices the integral operations are eliminated. (Mirzaee and Maleknejad,2006)

Adomian (1989; 1994) has used the decomposition method was used for solving the linear equations, the nonlinear equations, linear regression equations, partial regression equations etc. The equation is used for solving stochastic as well as non- stochastic equations. And at the same time the equation is also devoid of the problems of the perturbation, discretisation etc.

The applicability of the differential equations was introduced by Kaleva (1987). The comparison was 
done by Lopez and the solutions as numbers were found out by Friedman and Knadell.Allahviranloo was the person to apply and find solutions for the heatwaves which behave in the Fuzzy environment used the Hukuhara Differentiability.

Osman et al. (2020) used it to solve the wave patterns. The Taylors formula was used for this. The Adomian decomposition method and the Laplace modification is used to solve the nonlinear differential-integral equation. The equation was based on the Volterra Fredholm calculation. This was based on Caputo's derivative.

The non-linear function having the form of $\mathrm{N \mu}-\mathrm{g}(\mathrm{n})$ and $\mathrm{g}$ is defined as the smooth function then the Equation can be made into multiple Adomian Decomposition Equations which can be brough into a convergent solution. (Pang and Li, 2020)

The "Non-linear Shrodinger Equation" is used to solve a lot of problems in the data transmission in the fibre optics. At the same time the wide uses of the above has led to the extension of the equation using the Sine Cosine Method, Hirota's direct method etc. This can also be solved numerically. And this is made possible through the "Improved Adomian Decomposition Method. This will lead to more accuracy of the solution. (Bakodah et al. ,2020)

Several researches has paved way for the Adomian Decomposition Method improvisation. This was made possible through the adding a parameter which is already determined. The method used for this is Global Squared Residual Approximation. This will not only speed up the convergence but also increase the area of the convergence. (Turkyilmazoglu, 2019)

Bernstein Polynomial is used to modify and make the Adomian Decomposition Method efficient. There are also other method to solve the Adomian Decomposition Method. This include the homotopy perturbation, iteration, differential transform method, other iteration techniques etc. The working of this is proved in several situations involving the fluid properties like Maxwell fluid, Oldryoid B fluid etc. Three types of polynomials are used for the improvement of the Equation. They are Laguerre Polynomials, Chebyshev polynomials, Legendre Polynomials.

The Bernestein Polynomial is defined within the interval of $[0,1]$

$\mathrm{B}_{(\mathrm{i}, \mathrm{m})(\mathrm{X})}=(\mathrm{m}) \mathrm{x}^{\mathrm{i}}(1-\mathrm{x})^{(\mathrm{m}-\mathrm{i})}$

$$
\text { i }
$$

(Qasim and Rawi,2018)

Li and Pang (2020) states that the dire need of a method to describe any natural phenomena which has the preceding events and memory which will affect the present situation. In those cases, the mathematical modelling cannot be done with the help of the operators which are local. It can only be done by the non-local operators only.

Salawu and Sobamowo (2020) states that the dynamic vibration of a rectangular plate which have the is isotropic and the vibration is free is stated by the partial differential equations. This is again restated as the ordinary differential equations. Both are also non-linear in nature. This conversion is made possible through the Galerkin Separation Method, the ordinary differential equations are solved through the Adomian Decomposition Method. Werafalli and Aburuga has also used the similar method for solving the behaviours of the rectangular plates. This is very important in the field if engineering where the plates occupy a prominent role. They are also important for the safety purposes in their interaction with the fluids. The sum of the terms in the equation for applying the decomposition method. The Adomian Decomposition Method proved to be helpful to in reducing the complexity of different steps and different type of equation involved the homogenous and non- homogenous equations, linear and nonlinear equations, the equations with the coefficients which are variable as well as constant.

$$
\begin{gathered}
w^{\infty} \sum_{n=0}^{\infty} w_{n} \\
n=0
\end{gathered}
$$

The term which is non-linear in nature is converted into the equation

$$
\mathrm{N}(w)=\sum_{\mathrm{n}=0}^{\infty} \mathrm{A}_{\mathrm{n}}
$$

The principle behind this is $\mathrm{Lw}+\mathrm{Nw}+\mathrm{Rw}=g(x)$ If the order of the of the linear operator is 4 it is further defined as $\mathrm{L}=\mathrm{d}^{4} / \mathrm{dx}^{4}$

The $\mathrm{L}$ is the Linear Operator here, $\mathrm{N}$ is the nonlinear operator, $\mathrm{R}$ is the rest of the linear operators and the $\mathrm{g}(\mathrm{x})$ is the function which is inhomogeneous.

The research on the heatwaves which are fuzzy in nature also proves the use of the Adomian Decomposition Method. This is used along with the Variational Iteration Method. The differential equations which are fuzzy are converted into the crisp differential equations and the Variational Iteration Method (VIM) and the Adomian Decomposition Method (ADM) are used for the equations. Any number which is called by the name Fuzzy are two pairs of numbers. They can be 
denoted by $\mathrm{u}$ and $\mathrm{u}$ - and the functions are the functions of $\mathrm{u}(\mathrm{r})$ and $\mathrm{u}-(\mathrm{r})$

On the basis of the Zadeh's principle which is used for the extension of the differential equations the $\mathrm{u}$ $+\mathrm{u}-(\mathrm{x})=$ super $\min \{\mathrm{u}(\mathrm{y}), \mathrm{v}(\mathrm{x}-\mathrm{y})\} \mathrm{x} \mathcal{E} \mathrm{R}$

There are a lot of methods apart from the Adomian Decomposition Method to solve the non-linear systems. They are Kernel Algorithm method, the Sine Cosine method, the tanh method, homotopyperturbation method etc.

(Gong, Osman and Mustafa,2020)

\section{METHODOLOGY}

The methodology is just cross verifying the different applications of Adomian Decomposition Method in different fields. The paper reviews the researches based on the Adomian Decomposition Method and compares it usefulness in reference to other methods.

\section{FINDINGS, ANALYSIS AND INTERPRETATIONS}

The possibility of the Adomian Decomposition method in solving the Focker-Plank equation. The Adomian Decomposition Method solves it by using a method of convergent series of equations. This is done first by linearizing and then applying the decomposition method to it. But the Adomian Decomposition Method is not applicable in the cases of the high-indexed differential equations which are semi-explicit. Also, this is applied for hyperbolic and parabolic and differential equations which are dispersive in nature. Also, it is used to find out the heat conduction as well as the convection method.

This can be generalised to different situations and can be represented by the formula.

$\mathrm{u}-\Theta(\mathrm{u})=\mathrm{g}$ where $\mathrm{u}$ is the unknown which has to be found out and $\Theta$ is the linear operator. It is also used to find out the differential equations which arise from the calculus variations. (Tartari et al., 2007)

The stochastic equations are very much vulnerable to the small changes in the parameter of the inputs so that the outputs may be different. In this context Adomian Decomposition Method is very useful. It is also applicable in the case of the delay equation and the matrix equations. Since any model developed under this method is confined by time and space the boundaries must also be decided accordingly. And since small changes in the parameters will affect the model drastically in the case of the stochastic equations then there is difficulty in arriving at a convergent solution.

Here considers a thermodynamic system where the bounded system is the Surface $\mathrm{S}$ with volume V and the heat and the temperature of the system is represented by $\mathrm{u}(\mathrm{x}, \mathrm{y}, \mathrm{z}, \mathrm{t})$

The temperature Q1 $=\frac{\int_{t 1}^{t 2} \quad d t \iint k(x, y, z) \partial u}{\partial n} d S$

Here $\mathrm{K}$ is the heat conductivity and $\mathrm{n}$ is the normal to the surface $\mathrm{S}$. The solution to the above is found out by assuming that the surroundings are at uniform temperature and bound by the boundary condition of $\alpha(\mu-\mu \mathrm{m})$ (Adomian,1994).

The Adomian Decomposition Method is also used to work out the flow of the polar materials in a porous structure. The flow is due to the suction or injection pressure on the walls of the material. Also, the Adomian Decomposition method is applied after the nonlinear differential equations which are coupled and they are subjected the Berman's similarity transformation. After that the mas flow is solved through the Adomian Decomposition Method (Aski et. al.,2014).

Adomian Decomposition Method is also used to find out the solution for the Stefan problem. The Stefan problem is such that the homogenous medium of the temperature which is undergoing a phase change It is bounded by the temperature condition where the latent heat is absorbed and bound by those variables of the latent heat, It is the Newman Boundary, Dirichlet Boundary and Stefan boundary and Leibnitz equation is also used for this (Bougoffa et al.,2018).

Burgess equation calculates the proliferation of the cancer cells by the Adomian Decomposition Method (Burgess, 1997).

But the Adomian Decomposition Method will be failing if the iteration process leads to the divergence which will not provide a convergent solution. (Nelson,1988)

The applicability of the Adomian Decomposition Method is such that its applicable in a wide array of the situations: partial and ordinary differential equations, integro-differential equations, integral equations, Kawahara equations, Ricatti equations etc. The method of calculation is very simple which replaces other complex methods like Picard method. It can also accommodate any number of the unknown variables. It can also find solutions in the non- linear cases where iterative method fail to apply.

Homotopy Perturbation Method is very useful in the case of Adomian Decomposition Method also. It does not require the small parameters in the equation to be solved where a small parameter is already imbedded in the system of the equation. The imbedded parameter is defined as $\mathrm{p} \varepsilon[0,1]$ This method is invented by He (1998). The equation proposed by him is as follows

$$
\begin{aligned}
& \mathbf{Y}_{\text {app }}=\mathbf{Y 0}\left(\mathbf{X 0 )} \int_{0}^{X 0} \lambda(\mathrm{LYO}+\mathrm{NYO}) \mathrm{dx}\right. \\
& \lambda=\text { Lagrange multiplier }
\end{aligned}
$$


The Variational Iteration Method is more flexible to us as well as produce more precise results which can be again be used in combination with the Adomian Decomposition Method. (Zheng and Zhang, 2017)

\section{CONCLUSIONS}

The Adomian Decomposition Method is very useful for the lengthy computational differential Equations. The applicability increases because it is applicable for both Stochastic and Non-Stochastic or deterministic models. Also, the convergence is an essential perquisite. So, the divergent situation needs to devise a better computational method similar to the Adomian Decomposition Method. It has wide applicability in a variety of situations in the Physical, thermodynamics as well as other dynamic systems. The Adomian Decomposition Method can be made use of in the analysis of the stock market and more volatile capital structural decision-making situations in business also where linearity does not apply. Its uses in relation to other methods will make it a still more effective tool than using it in isolation.

\section{REFERENCES}

[1] Adomian, G., \& Rach, R. (1985). On the solution of algebraic equations by the decompoistion method. J Math. Anal., Appl.Vol 105, pp 141-166.

[2] Adomian, G. (1989). Comments on a counter example to decomposition. Journal of Computational and Applied Mathematics., Vol 26, pp 375-376.

[3] Adomian, G. (1994). Solving Frontier problems of Physics: The Decomposition Method.,. International Journal of Mathematical and Computer Modelling. USA: Springer Science

[4] Ashyralyev, A \& Hicdurmaz, B. (2011). A note on the Schrodinger differential equations. Kybernetes., Vol 40(5/6), pp 736-750.

[5] Aski, S.F., Nasirkhani, S.J., Asgari, M. A. (2014). Application of Adomian Decomposition Method for Micropolar flow in a porous channel. Journal of Propulsion and power Research., Vol 3(1), pp 15-21.

[6]Bakodah, H.O, Banaja, M.A. ,Alshaery,A.A. \& Qarni A.1,.A.A (2020).Numerical Solutions of Dispersive Optical Solitions with Schrodinger Hirota Equation by Improved Adomian Decomposition Method .,Vol 2019, Article Id 2960912..https://doi.org/10.1155/2019/2960912

[7] Bougoffa, Randolph, R, Duan, W.A.J. (2015). On the Adomian decomposition method for solving the Stefan problem. International Journal of Numerical Methods for Heat and Fluid Flow, Vol 25(4), pp912-928.
[8] Burgess, P.K., Kulesa, P.M., Murray \& J. D, Alvord Jr. E.C. (1997). The Interaction of growth rates and diffusion coefficients in a threedimensional mathematical model of gliomas. Journal of Neuropathology and Experimental Neurology 56, pp 704-713.

[9] Cherruault, Y., \& Mora, G. (2005) . Optimisation Globale: The Orie des Courbes alpha-denses. Economica.Paris:.Craik, A.D.D.

[10] He, J.H (1998). Approximate analytical situation for seepage flow with fractional derivatives in porous media. Journal of Computational Methods applications in Mechanical Engineering., Vol167., pp 57-68.

[11] He, J.H (2020) Generalised variational principles for bucking analysis of circular cylinders. Acta Mech.Vol 231., pp 899-906.

[12] Kaleva, O. (1987) Fuzzy Differential Equations. Fuzzy sets SystVol 24, pp 301-317.

[13] Maleknejad, K. \& Mirzaee, F. (2006). Numerical solution of integro-differential equations by using rationalized Haar functions method. Kybernetes, Vol. 35(10), pp.1735-1744.

[14] Nelson, P. (1988). Adomian Method of Decomposition: critical review and examples of failure. Journal of Computational and Applied Mathematics., Vol23, pp 389-393.

[15] Osman, M., Gong, Z., and Mustafa, A.M. (2020). Comparison and fuzzy Adomian decomposition method with fuzzy VIM for solving fuzzy heat-like and wave -like equations with variable coefficients., Advances in Differential Equations. Article 327. https://doi.org/10.1186/s13662-020-02784-w

[16] Qasim, A.F., Alrawi, E.S. (2018). Adomian Decomposition Method with modified Bernstein Polynomials for solving ordinary and partial differential equations. Journal of Applied Mathematics. https://doi.org/10.1155/2018/18031 07

[17] Turkyil, M. (2019). Accelerating the Convergence of the Adomian Decomposition Method. Journal of Computational Science.Vol 31.pp54-59.

[18] Tartari M., Dehghan M. \& Razzaghi, M. (2007) Application of the Adomian Decomposition Method for the Focker-Planck Equation. Mathematical and Computer Modelling.Issue45, pp639-60.

[19] Osman, M., Gong, Z., \& Mustafa, A.M. (2020). Comparison of fuzzy Adomian Decomposition Method with Fuzzy VIM for solving fuzzy heat-like and wave-like equations with variable coefficients. Adv Differ Equ.pp 327.

[20] Pang, Y., \& Li, W. (2020). Application of Adomian decomposition method to non-linear Systems. Advances in differential Equations. 
Article 67. https://doi.org/10.1186/s13662-0202529-y.

[21] Sobamowo, G.M., Salawu, S.A. \& Yinusa, A.A. (2020). Application of Adomian Decomposition method to free vibration analysis of thin isotropic rectangular plates submerged in fluid. J Egypt Math Soc.Vol 28 https://doi.org/10.1186/s42787-020-0071-4.

[22] Werfalli, N.M. \& Aburuga, T.K. (2005). Galerkin finite element analysis for bending of anisotropic plates. Fourth Arab. Conference. Material Sciences. ACMS Tripoli Libya 4(1) Pp 319-331.

[23] Zheng, L. \& Zhang, X. (2017). Modelling and Analysis of Modern Fluid Problems. Mathematics in Science and Engineeering.Acdemic Press.pp 137.

[24] Zitoun, F. B, Cherruault, Y. (2010). A method of solving nonlinear differential equations. Kybernetics. Vol39(4), pp 578-597. 